Article

\title{
The Complete Chloroplast Genomes of Two Lespedeza Species: Insights into Codon Usage Bias, RNA Editing Sites, and Phylogenetic Relationships in Desmodieae (Fabaceae: Papilionoideae)
}

\author{
Yamuna Somaratne ${ }^{1}\left(\mathbb{D}\right.$, De-Long Guan ${ }^{1}$, Wen-Qiang Wang ${ }^{2}$, Liang Zhao $^{3}$ and \\ Sheng-Quan Xu ${ }^{1, *(D)}$ \\ 1 College of Life Sciences, Shaanxi Normal University, Xi'an 710062, China; yamuna@snnu.edu.cn (Y.S.); \\ guandelong@snnu.edu.cn (D.-L.G.) \\ 2 College of Life Sciences, Yan'an University, Yan'an 716000, China; wenqiangwang@yau.edu.cn \\ 3 College of Life Sciences, Northwest A \& F University, Yangling 712100, China; biology_zhaoliang@126.com \\ * Correspondence: xushengquan@snnu.edu.cn
}

Received: 24 November 2019; Accepted: 30 December 2019; Published: 31 December 2019

\begin{abstract}
The genus Lespedeza (tribe: Desmodieae) consists of about 40 species that have high medicinal and economic value. However, in this genus, using morphological characters, the species identification is quite complicated, which can be solved by the analysis of the complete chloroplast genomes. As primary organelle genomes, the complete genome sequences of chloroplasts (cp) provide unique molecular information to study the divergence of species, RNA editing, and phylogeny. Therefore, to the best of our knowledge, for the first time, we sequenced the complete cp genomes of two representative Lespedeza species: Lespedeza davurica and Lespedeza cuneata. The cp genomes of both the species were found to be $149,010 \mathrm{bp}$ in length, exhibiting the typical angiosperm chloroplast structure containing four regions. The Lespedeza cp genomes showed similar conserved gene contents, order, and orientations with a total GC content of $35.0 \%$. A total of 128 genes, including 83 protein-coding genes, 37 tRNAs, and eight rRNAs, were identified from each genome. Unique molecular features of the two Lespedeza cp genome sequences were obtained by performing the analysis of repeats, sequence divergence, codon usage, and predicting the RNA editing sites in addition to phylogenetic analysis with other key genera in tribe Desmodieae. Using the two datasets, the phylogenetic relationship of Lespedeza species among Deasmodieae was discovered, suggesting that whole cp genomes provided useful information for phylogenetic studies of these species.
\end{abstract}

Keywords: Lespedeza; desmodieae; papilionoideae; fabaceae; chloroplast genome; phylogenetic relationships

\section{Introduction}

Lespedeza is a genus in the tribe Desmodieae, which belongs to the subfamily Papilionoideae of the family Fabaceae, and this genus comprises nearly 40 species distributed mainly in eastern Asia and eastern North America [1]. These plants grow as shrubs or trailing vines and can be either annual or perennial. Due to the structure of the plants and their inflorescences, some species like Lespedeza bicolor, are used as ornamental plants. The leaves of these plants can be used as forage and for soil enrichment, and its deep root system is useful to prevent soil erosion. For example, Lespedeza cuneata and Lespedeza davurica plant as a forage crop for livestock and are used to conserve soil and improve soil conditions [2,3]. Due to its high tannin content at maturity, animals are fed on L. cuneata at an early stage of the plant. The high adaptability to drought and good forage quality of L. davurica are 
important for its use as a fodder legume [4]. Similar to most other legumes, the deep root system of the Lespedeza has nitrogen-fixing nodules that harbor the symbiotic soil bacteria, making this plant a nitrogen fertilizer [5]. Moreover, several bioactive compounds that have antidiabetic activities have been extracted from some species of Lespedeza that were used in medicine to prevent diabetes in ancient times. Recent studies have shown that $L$. davurica exerts a protective function on $\beta$-cells in the pancreas, which can regulate blood glucose in Type 1 diabetes [6]. Due to the distinct values of each Lespedeza species, it is vital to differentiate them at the species level for proper utilization and conservation.

Classification of these Lespedeza species has been performed for many years using different methods. Ohashi [7] classified genus Lespedeza into two subgenera, Lespedeza and Macrolespedeza, based on the floral characters and the pollen morphology [8]. Macrolespedeza is distributed only in East Asia while Lespedeza is distributed in both eastern North America and East Asia. The identification of eastern Asian and eastern North American species based on morphological characteristics was discussed decades ago [9]. However, Ohashi and Nemoto (2014) [10] showed that these two subgenera differ only in seedling morphology. Phylogenetic analysis has been performed from the DNA sequences of the nuclear ribosomal internal transcribed spacer (ITS) and plastid regions of Lespedeza species [1]. Moreover, Kim et al. (2012) [11] classified Lespedeza species using their metabolite profiling; previous research has shown that the floral morphology of Lespedeza was similar to that of two genera, Campylotropis and Kummerowia, which were formerly placed in Lespedeza [8]. Therefore, an accurate method of plant identification is essential for Lespedeza species. The whole chloroplast genome contains more effective molecular markers, referred to as DNA barcodes, which are useful for identifying species accurately. Chloroplast genomic sequences provide molecular information that is a good resource for plant systematics, phylogenetic studies, and population genomics [12,13]. Therefore, in recent years, many chloroplast genomes in the family Fabaceae, have been completely sequenced. Complete chloroplast sequences of L. maritima, classified in subgenus Macrolespedeza of the genus Lespedeza, has been reported recently [14].

The typical angiosperm chloroplast genome is a circular, double-stranded DNA molecule with a size ranging from 120 to $160 \mathrm{~kb}$ [15]. It contains about 130 genes within the regions of two identical copies of inverted repeats (IRs), a large single-copy (LSC) region, and a small single-copy (SSC) region $[16,17]$. Chloroplast genomes are maternally inherited, and they contain highly conserved gene structures [18]. Because of its stable structures and uniparental inheritance, it has been used in comparative analysis to understand the evolution of closely related species. Over the past few years, whole chloroplast (cp) genome sequencing with next-generation sequencing technology (NGS) has become much more accurate, less expensive, less labor-intensive, and less time consuming than conventional Sanger sequencing [19-21]. Illumina-based NGS technology has been widely used recently, and the molecular structures of many plant $\mathrm{cp}$ genomes have been discovered in detail within a short period [22]. Chloroplast genomic information has been widely used for molecular marker development in different evolutionary studies $[17,23]$. Polymorphic simple sequence repeats (SSR) identified in the cp genomes were previously used for species identification, population genetics, and phylogenetic studies [24,25]. Codon usage bias in cp genomes has been studied as it plays an important role in gene expression [26,27]. Furthermore, identifying the RNA editing sites in the cp genome is vital to understand the correct translation process and mutations of genes [28,29]. Comparative analyses of $\mathrm{cp}$ genomes based on these variations are useful resources to investigate the evolutionary relationship among species.

In this study, we obtained the complete cp DNA sequences of two Lespedeza species in subgenus Lespedeza, Lespedeza davurica and Lespedeza cuneata, using an Illumina HiSeq X Ten sequencing platform. Our aims of this study were (1) to explore the molecular features of each genome compared them with the Macrolespedeza species, L. maritima, and other members of the tribe Desmodieae; (2) to observe the variable regions between species in the genus Lespedeza; (3) to examine the SSR distributions, codon usage patterns, and RNA editing sites among Lespedeza cp genomes; and (4) to determine phylogenetic 
position of subgenus Lespedeza in the tribe Desmodieae. The results obtained from this study will provide a deeper understanding and classification of this genus in tribe Desmodieae.

\section{Results}

\subsection{Comparison of Lespedeza cp Genome Structures}

The length of the complete genomes of both L. davurica (MH800328) and L. cuneata (MN268503) were $149,010 \mathrm{bp}$ and exhibited the typical angiosperm circular chloroplast structure containing four regions: Large single-copy region (LSC; 82,373 bp, 82,395 bp), small single-copy region (SSC; 18,975 bp, $18,951 \mathrm{bp})$, and a pair of inverted repeats (IR) each $(23,831 \mathrm{bp}, 23,832 \mathrm{bp})$. The cp genomes showed similar gene contents, orders, and orientations (Figure 1). Nearly $52.2 \%$ of the complete chloroplast genomes consisted of protein-coding genes (77,868 bp), 6.1\% consisted of rRNAs (9062 bp), and 1.8\% consisted of tRNAs (2794 bp) in both species. In addition, similar characteristic features of the $\mathrm{cp}$ genomes were shared between L. davurica and L. cuneata.

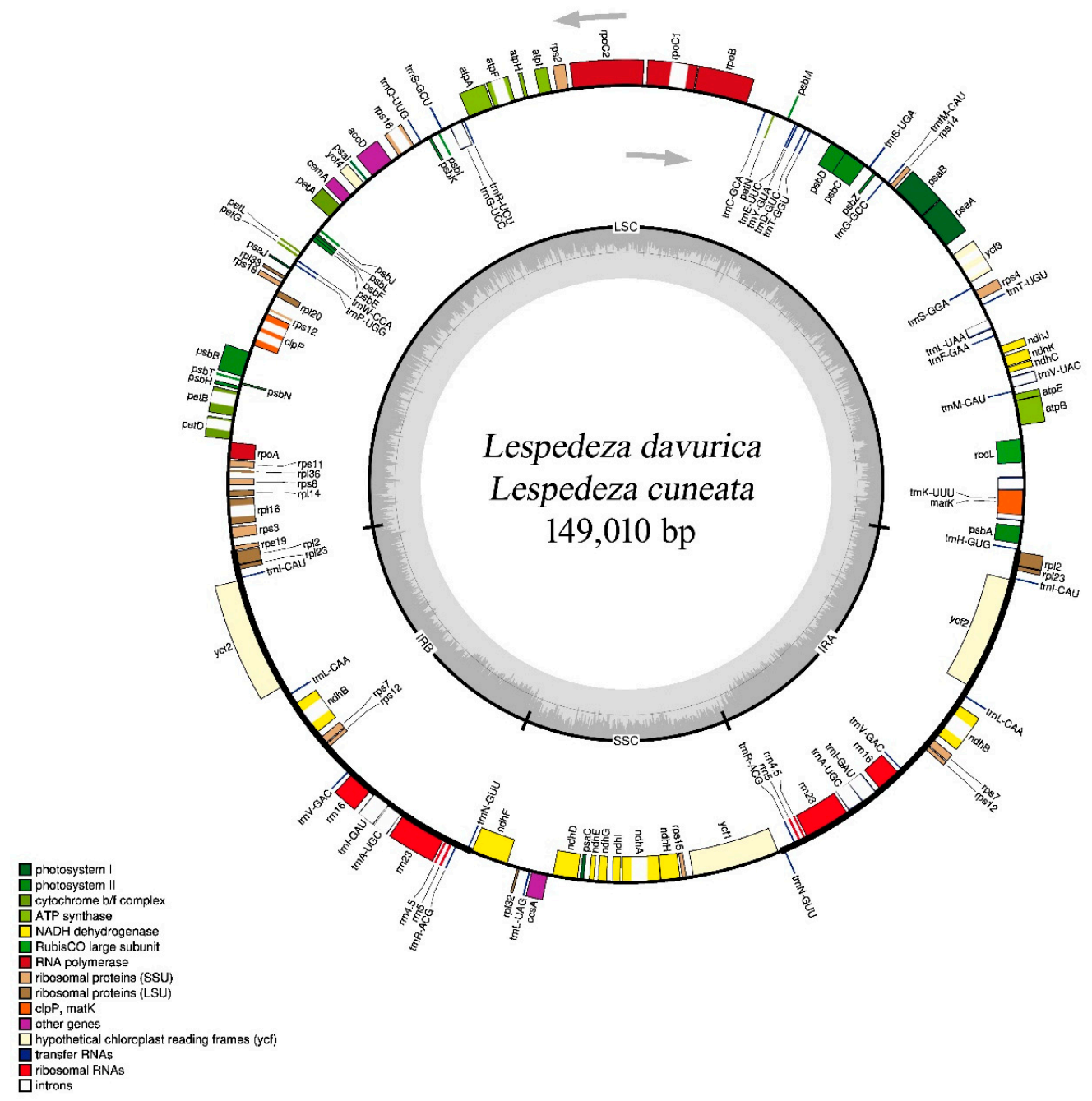

Figure 1. Gene map of Lespedeza davurica and Lespedeza cuneata chloroplast genomes. Genes on the inside of the map are transcribed in the clockwise direction and genes on the outside of the map are transcribed in the counterclockwise direction. The darker gray in the inner circle represents GC content whereas the light gray corresponds to AT content. Different functional groups of genes are shown in different colors. The figure was drawn with OrganelleGenomeDRAW [30]. 
The analysis revealed that the GC content of the cp genome of both the species was $35.0 \%$ and the IR regions exhibited highest GC content (42.2\%) followed by the LSC regions (32.5\%) and SSC regions $(28.1 \%)$. The coding sequences (CDS) showed a similar total GC content of $36.0 \%$ in both species (Table 1).

Table 1. Summary of chloroplast genome features of Lespedeza.

\begin{tabular}{ccc}
\hline Characteristics & Lespedeza davurica & Lespedeza cuneata \\
\hline Accession Number & MH800328 & MN268503 \\
Total cpDNA Size & 149,010 & 149,010 \\
LSC & 82,373 & 82,395 \\
IR & 23,831 & 23,832 \\
SSC & 18,975 & 18,951 \\
Total CDS Length & 77,868 & 77,868 \\
Total tRNA Length & 2794 & 2794 \\
Total rRNA Length & 9062 & 9062 \\
Total Number of Genes & 128 & 128 \\
Coding Genes & 83 & 83 \\
rRNA Genes & 8 & 8 \\
tRNA Genes & 37 & 37 \\
Total GC $\%$ & 35.0 & 35.0 \\
LSC & 32.5 & 32.5 \\
IR & 42.2 & 42.2 \\
SSC & 28.1 & 28.1 \\
CDS & 36.0 & 36.0 \\
\hline
\end{tabular}

LSC: Large single copy, IR: Inverted repeat, SSC: Small single copy, CDS: Coding sequences, GC: Guanine and Cytosine.

A total of 128 total genes, including 83 protein-coding genes, 37 tRNAs, and eight rRNAs were identified from each genome and annotated using Dual Organellar GenoMe Annotator (DOGMA). In addition, 16 functional genes, including five protein-coding genes $(r p l 2, r p l 23, y c f 2, n d h B$, and rps7), seven tRNA genes (trnI-CAU, trnN-GUU, trnR-ACG, trnA-UGC, trnI-GAU, trnV-GAC, and $\operatorname{trnL}-C A A)$, and four rRNA genes ( $r r n 16, r r n 23, r r n 4.5$, and $r r n 5)$ were duplicated in the IR regions of each chloroplast genome.

Eight protein-coding genes (petB, petD, atpF, ndhB, ndhA, rpoC1, rpl16, and rps16) and eight tRNA genes contained one intron, while three protein-coding genes $(r p s 12, y c f 3$, and $c l p P)$ contained two introns (Supplementary Materials, Table S1). The $5^{\prime}$ end of the rps12 gene was located in the LSC region and $3^{\prime}$ end duplicated in the IR regions in both the species. The basic features of these two chloroplast genomes were compared to six other species in the tribe Desmodieae: Lespedeza maritima, Kummerowia striata, Campylotropis macrocarpa, Hylodesmum podocarpum, Desmodium heterocarpon, and Ohwia caudata (Table S2).

\subsection{Codon Usage Bias and Prediction of RNA Editing Sites}

We analyzed the codon distribution among all protein-coding genes in the two Lespedeza species and compared them with L. maritima. These protein-coding sequences represented a total of 25,956 codons in L. davurica and L. cuneata each and 25,958 codons in L. maritima. They belonged to 61 codon types, which encoded 20 amino acids. Leucine was the most abundant amino acid, whereas cysteine showed the least abundance in three species (Figure 2). The most often used synonymous codon was AAA, encoding lysine, and the least used was CGG, encoding arginine (Table S3). Relative synonymous codon usage (RSCU) is a good indicator to measure codon usage bias in coding sequences. This value is the ratio between the observed frequency and expected frequency of a codon. When the use of synonymous codons is less frequent than expected, the RSCU value is less than 1 . However, when this value is more than 1, the expected frequency is less. Methionine and tryptophan showed RSCU $=1$, indicating that codons AUG and UGG had no bias and preferences. The highest RSCU value was 
for UUA ( 2.06) in leucine, and the lowest was GGC (0.3) in glycine. Moreover, leucine showed A or T (U) bias in all synonymous codons: UUA, UUG, CUU, CUC, CUA, and CUG. It was observed that the RSCU value for the specific amino acid increases with the number of codons. Leucine, serine, and arginine preferred six codon types, while methionine and tryptophan preferred one codon type. Similar codon usage patterns were exhibited in the three Lespedeza species (Table S3).

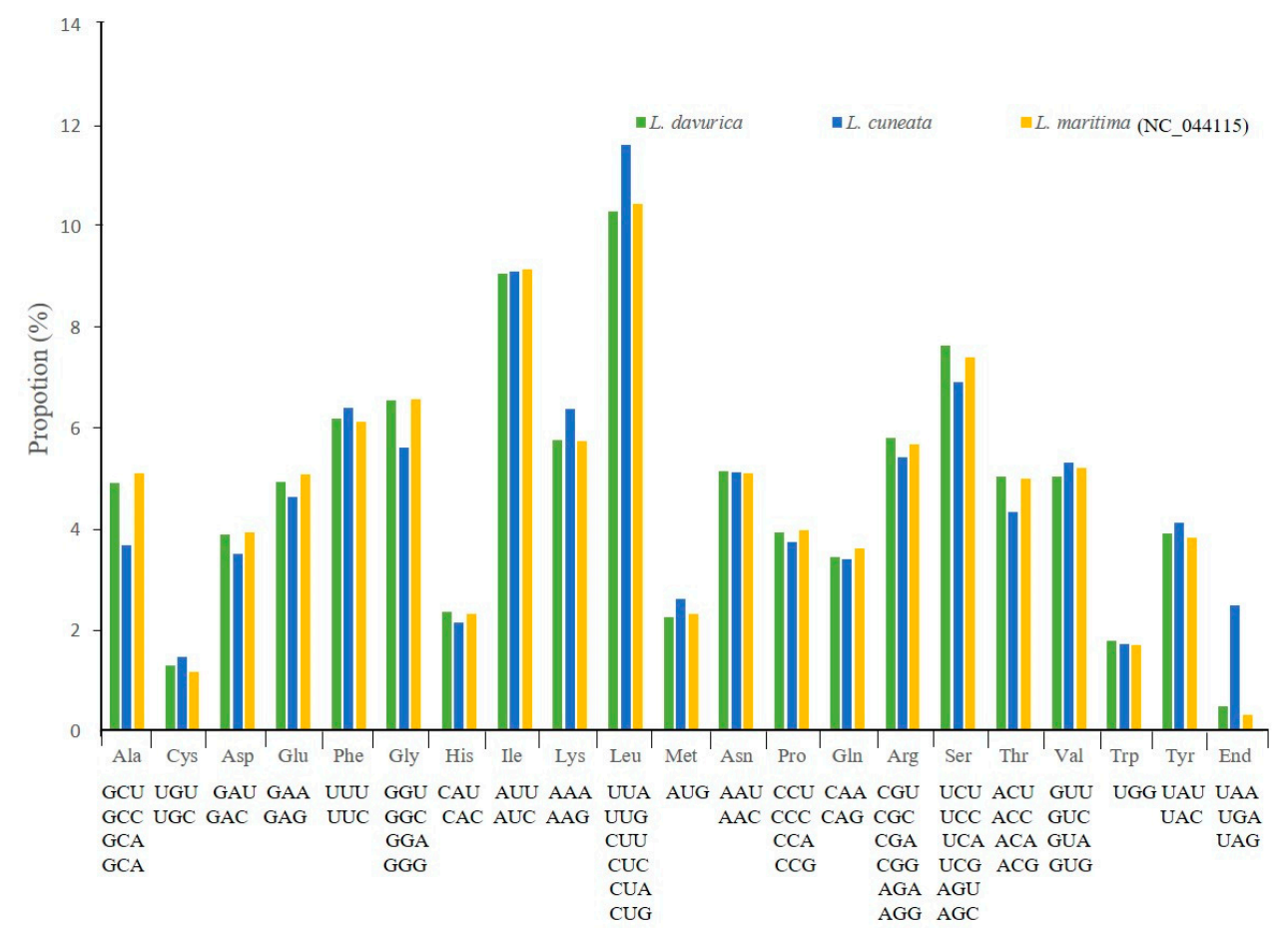

Figure 2. Amino acid frequencies of the chloroplast genomes of Lespedeza species.

All RNA editing sites were in the same nucleotide and amino acid positions in Lespedeza species. A total of 84 RNA editing sites were predicted in 35 protein-coding genes in cp genomes. All the codon changes were $C$ to $T$ conversions. The most editing sites were observed for $n d h$ genes. The $n d h B$ gene showed the most (13) editing events. Among the $n d h$ genes, $n d h B$ showed maximum editing events (13), followed by $n d h D$ (12), $n d h F(11) n d h A$ (3), and $n d h G$ (3). The most abundant amino acid change was the $\mathrm{S}$ to $\mathrm{L}$, with 18 out of 84 editing events (Figure 3). Editing was observed at first (21) and second (63) codon positions. The $\mathrm{H}$ to $\mathrm{Y}, \mathrm{P}$ to $\mathrm{S}$, and $\mathrm{L}$ to $\mathrm{F}$ amino acids conversions were due to changes in first codon position, while the $\mathrm{P}$ to $\mathrm{L}, \mathrm{S}$ to $\mathrm{L}, \mathrm{T}$ to $\mathrm{M}, \mathrm{A}$ to $\mathrm{V}, \mathrm{T}$ to $\mathrm{I}$, and $\mathrm{S}$ to $\mathrm{F}$ changes occurred due to second codon positions (Table S4).

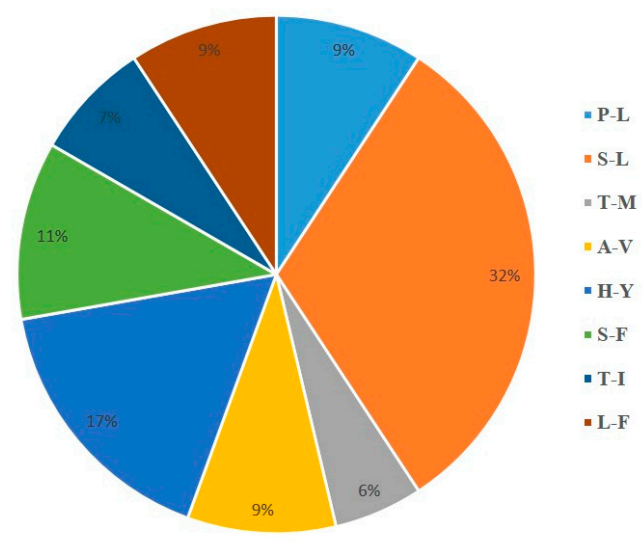

Figure 3. Types and percentage of amino acid exchanges arising from chloroplast RNA editing in Lespedeza. 


\subsection{Simple Sequence Repeats and Repeat Structure Analysis}

Simple-sequence repeats (SSRs), also known as microsatellites, are short tandem repeat DNA sequences that consist of repeating 1-6 nucleotide motifs distributed throughout the chloroplast genome [31]. It shows a high degree of polymorphism that can be used as a molecular marker in species identification [32]. A total of 75, 77, and 78 SSRs were detected in L. davurica, L. cuneata, and L. maritima cp genomes, respectively (Figure 4A; Table S5). Mononucleotide repeat A/T was the most abundant (Figure 4B) and was encountered 42, 41, and 44 times in L. davurica, L. cuneata, and L. maritima, respectively. The numbers of dinucleotide SSRs $(24,23$, and 22, respectively) were more than those of tetranucleotide SSRs, which were nine in each species. No pentanucleotide SSRs existed in these three species, and no trinucleotide SSRs were present in L. davurica and L. maritima. A hexanucleotide repeat was identified only in L. maritima. SSRs were distributed in LSC, SSC, and IR regions, with an abundance in the LSC as compared to the SSC and IR regions.

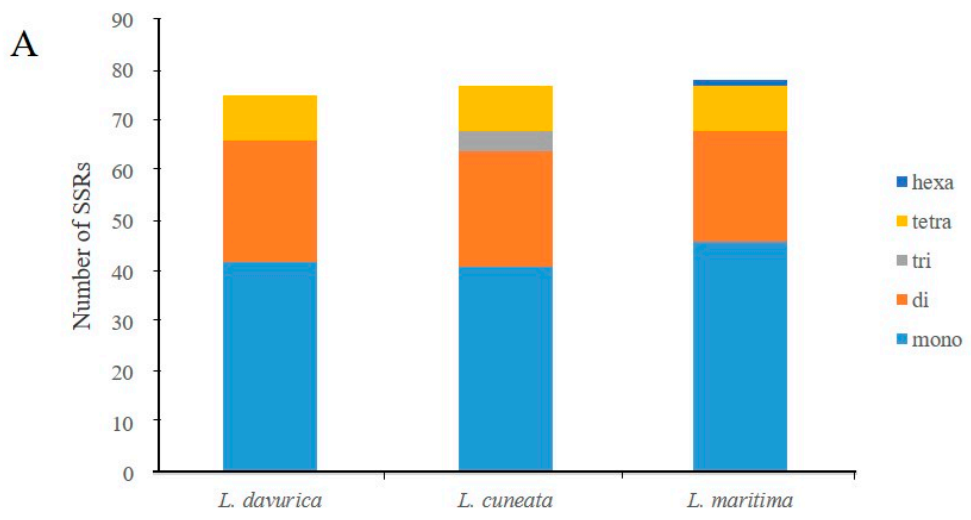

B

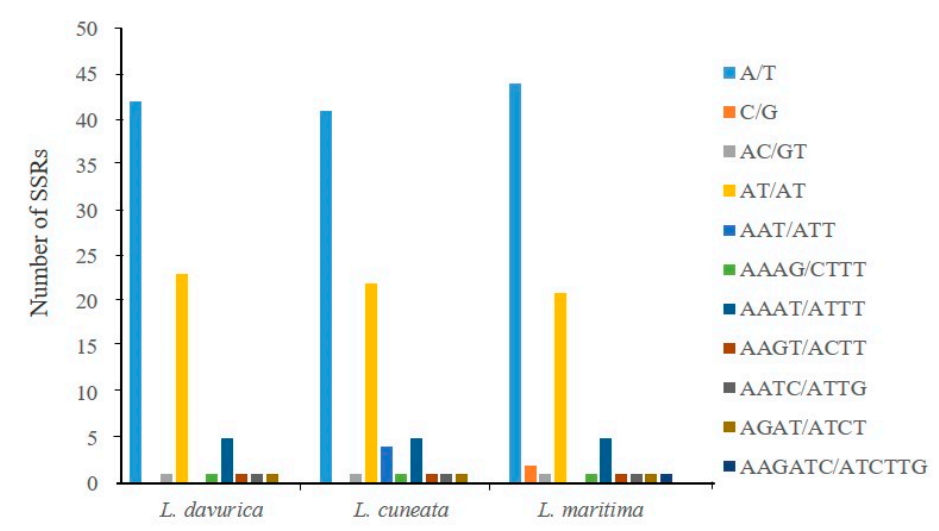

Figure 4. Distribution of simple sequence repeats (SSRs) in the cp genomes of Lespedeza. (A) Type of SSRs in the chloroplast genome of three species. (B) Distribution of SSRs in the chloroplast genome of three species.

Dispersed repeat sequences occurred in multiple copies of the genome, and they are recognized as a source of genomic variation and rearrangement [33]. This generates diversity among genomes in a population, which is useful for studies on the phylogenetic relationships among different genomes. Most of these repeats are distributed in the intergenic spacer (IGS) and intron sequences. The structure analysis of repetitive sequences revealed an equal number of repeats in the three Lespedeza chloroplast genomes with slightly altered repeat types. The repeats in L. davurica were comprised of 18 forward, 30 palindromic, and one reverse repeats, L. cuneata contained 18 forward, 27 palindromic, three reverse, and one complement repeats, while L. maritima consisted of 20 forward repeats, 27 palindromic repeats, one reverse, and one complement repeats (Figure 5A). The palindromic type was the most abundant 
repeat, with lengths in the range of 20-40 bp in all the three species (Figure 5B). These repeats could provide valuable information for studying the population phylogeny of these three species.

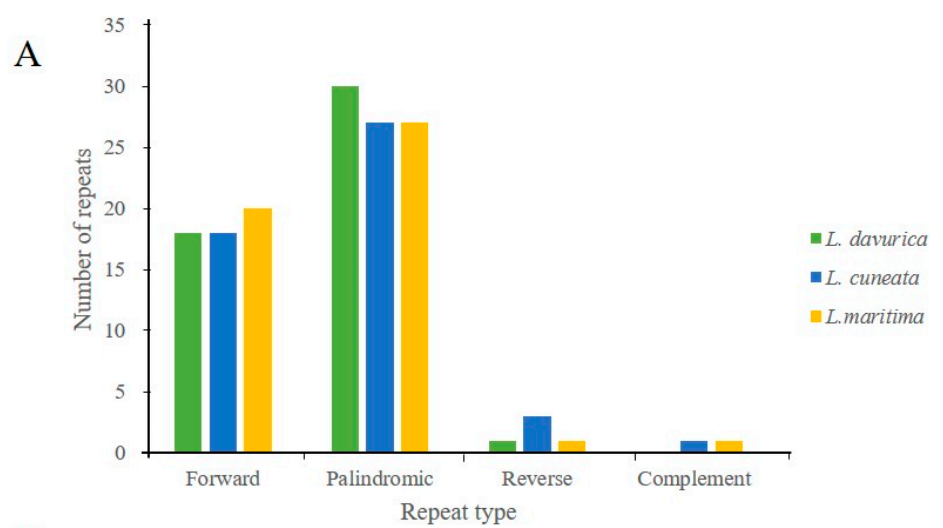

B

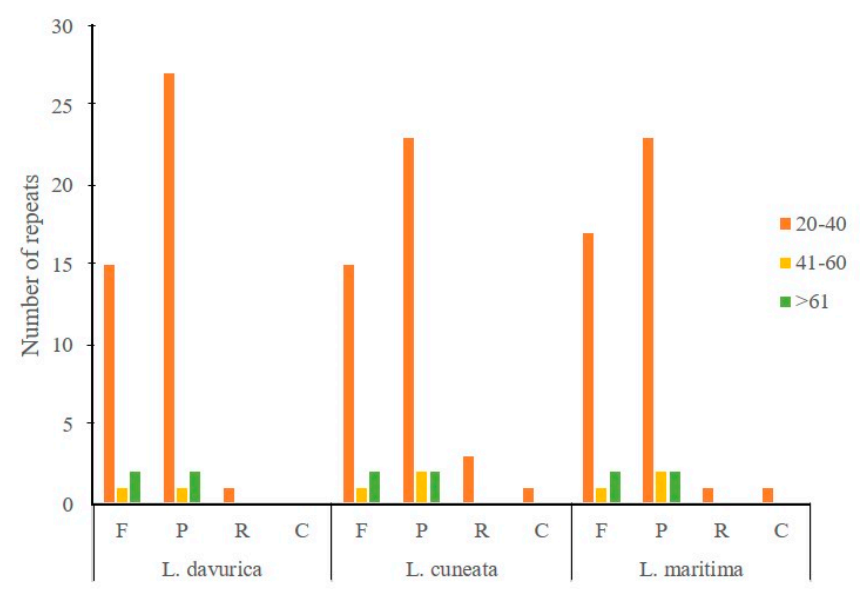

Figure 5. Repeat structure analysis of three Lespedeza sps. (A) Number of different types of repeats identified from the cp genomes. (B) Frequency of repeat lengths observed for different repeats. F, Forward; P, Palindromic; R, Reverse; C, Complement.

\subsection{Sequence Divergence Analysis}

mVISTA was used to compare whole chloroplast genome sequences of subtribe Lespedezinae including L. cuneata, L. maritima, Kummerowia striata, and Campylotropis macrocarpa, with L. davurica as a reference (Figure 6). Sequence divergence was very low among the species, suggesting a rather conserved cp genome. However, high nucleotide variations were observed between the three Lespedeza sequences for the protein-coding region $r p l 32$, and intergenic regions such as $\operatorname{trnL}-\operatorname{trn} T$, atpA-trnR-trnG, $n d h F-r p l 32$, and rpl32-trnL. Sliding window analysis results revealed the same variable regions in the cp genomes of the three Lespedeza species. The sequence divergence level was estimated through the calculation of nucleotide variability $(\mathrm{Pi})$ using the DnaSP software, and the average value of $\mathrm{Pi}$ among the Lespedeza species was estimated to be 0.00147 (Figure 7A). Two highly variable regions, atpA-trnR-trnG and ndhF-rpl32-trnL, were observed with the higher Pi values (>0.01) and were located at the LSC and SSC regions, respectively. Similar results were also obtained from mVISTA. However, the average Pi value of 0.00826 among all species in Lespedezinae (Figure 7B) indicated the existence of divergence between cp genome sequences of different genera. The loci $t r n K-r b c L, p s b M-p e t N, r p s 16-a c c D$, $r p s 3-r p s 19$, and ndhF-rpl32-trnL showed variability in both analyses, and $r p s 16-a c c D$ and ndhF-rpl32-trnL showed a remarkably higher Pi value ( 0.033) among all species. 

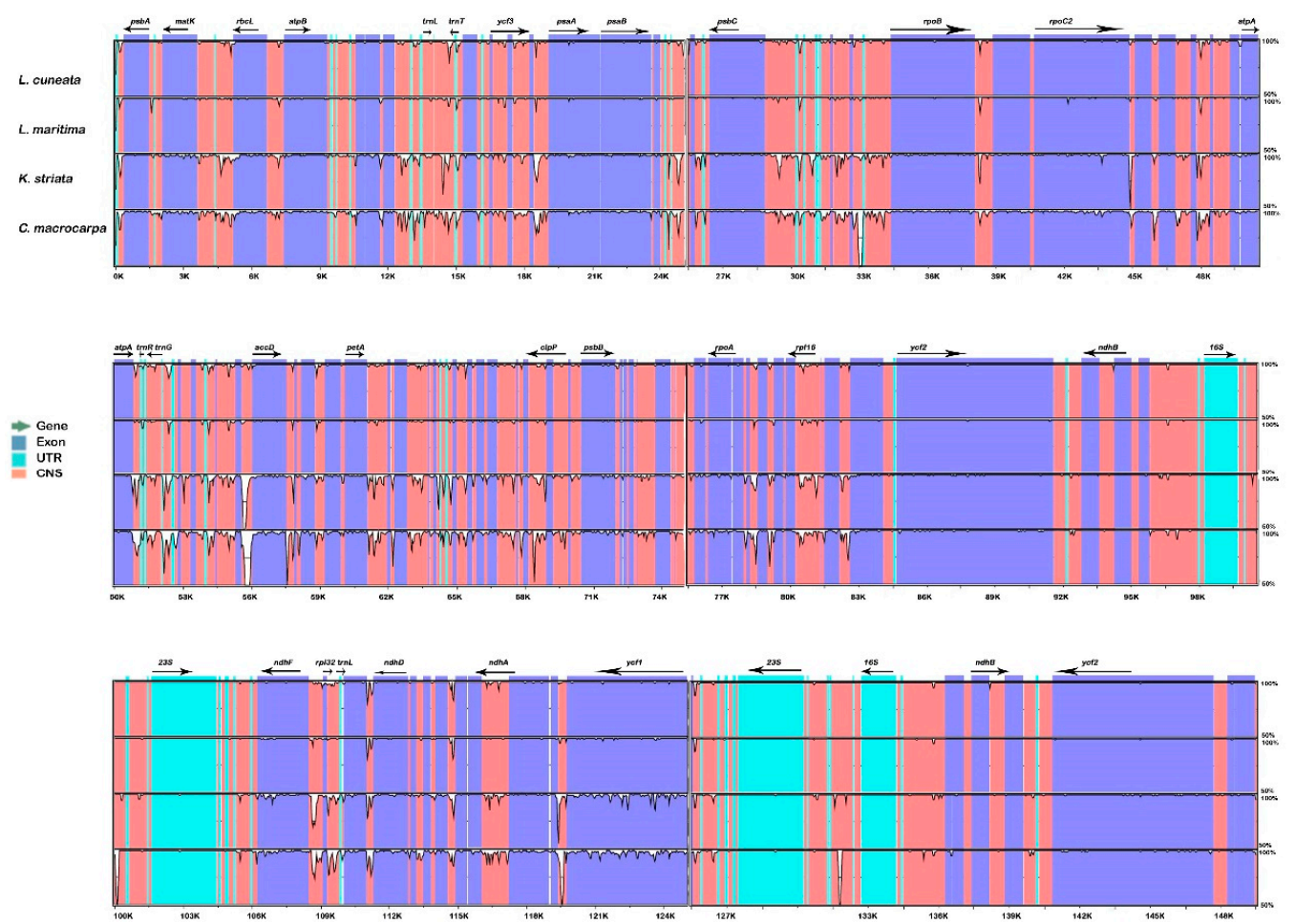

Figure 6. Sequence alignment plot comparing five cp genomes with L. davurica as a reference. Genome regions are color coded as protein coding, rRNA coding, tRNA coding, or conserved noncoding sequences. The vertical scale indicates the percentage identity, ranging from $50 \%$ to $100 \%$.

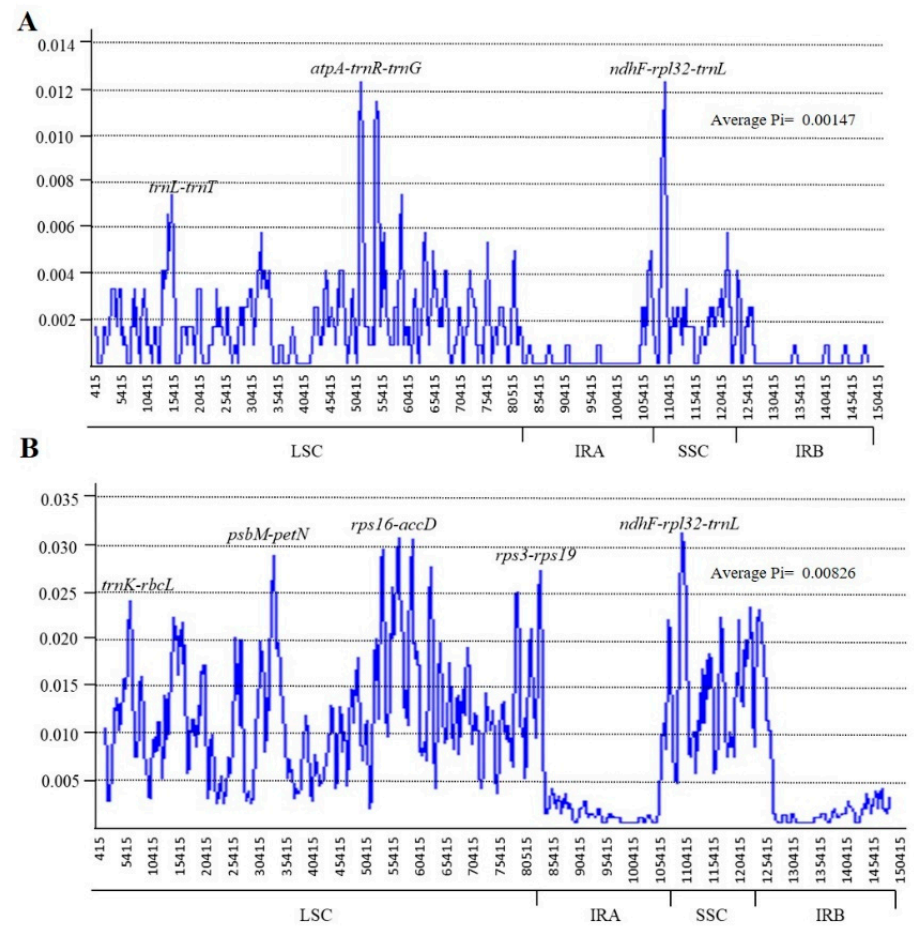

Figure 7. Sliding window analysis of the whole chloroplast genomes. (A) Pi among Lespedeza species. (B) Pi among species in Lespedezinae. Window length: 800 bp; step size: 200 bp. X-axis: Position of the midpoint of a window. Y-axis: Nucleotide diversity of each window. 


\subsection{Phylogenetic Analysis}

ML and BI trees showed similar results and identical topologies for both the datasets comprising the whole chloroplast genome sequences of different species of Desmodieae (Figure 8A) and the 68 protein-coding genes (Figure $8 \mathrm{~B}$ ). Support values in the ML tree were $100 \%$ in both datasets, and a sister relationship between L. davurica and L. cuneata was observed; moreover, both showed a sister relationship with L. maritima. Furthermore, Lespedeza formed a clade with Kummerowia striata and Campylotropis macrocarpa indicating their close relationship in the subtribe Lespedezinae. The high bootstrap and posterior probability values in this analysis suggested that the protein-coding genes and whole chloroplast genomes could be useful to find the phylogenetic positions and relationships of Lespedeza in the tribe Desmodieae.

A

B

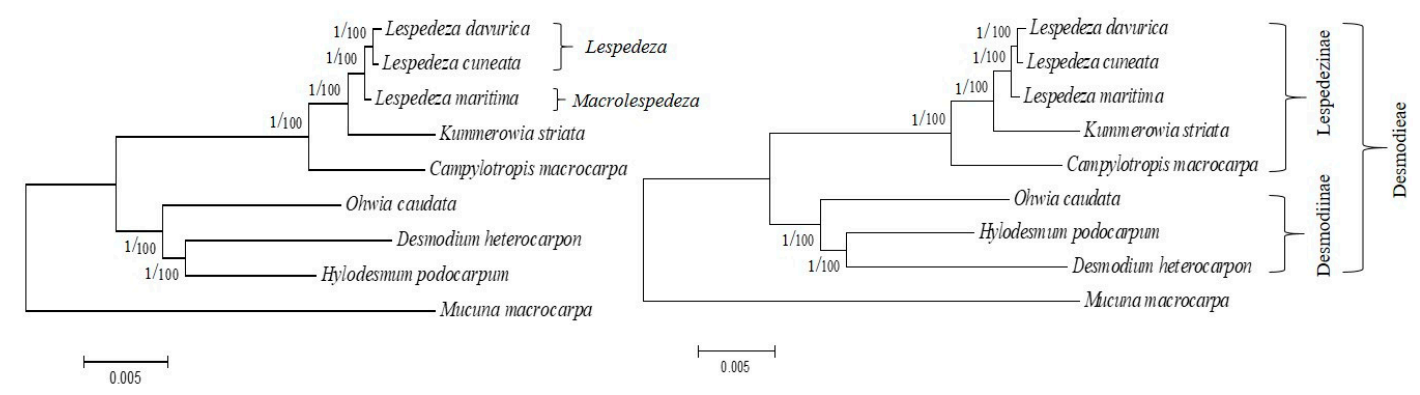

Figure 8. Phylogenetic relationship of Desmodieae species using maximum likelihood (ML) and Bayesian inferences (BI) analyses based on (A) whole chloroplast genomes, (B) 68 protein coding genes. The numbers in each node indicate ML bootstrap values on the right, and values on the left indicate Bayesian posterior probabilities (PP).

\section{Discussion}

The Papilionoideae is a subfamily of Fabaceae that is the third-largest to the Asteraceae and Orchidaceae families in angiosperms. Many economically important trees and shrubs belong to this family, which comprises about 770 genera and 19,500 known species [34,35]. Among them, Astragalus, Acacia, Indigofera, Crotalaria, and Mimosa are the most abundant genera in this family, and they include over 3000, 1000, 700, 700, and 500 species, respectively. According to the APG III plant classification system, these genera belong to six subfamilies, Cercidoideae, Detarioideae, Duparquetioideae, Dialioideae, Caesalpinioideae, and Papilionoideae, within family Fabaceae. Papilionoideae is a widely distributed subfamily comprising about 14,000 species from 503 genera, which belong to several tribes. The Desmodieae is one of the tribes in Papilionoideae and comprises about 32 genera [36,37]. Furthermore, this tribe has historically divided into subtribes such as Bryinae, Desmodiinae, and Lespedezinae [38]. In the Lespedezinae, a close relationship between three genera Campylotropis, Kummerowia, and Lespedeza has been discussed previously using the floral characteristics [8].

About 40 species listed for the genus Lespedeza include eastern Asia species and North American species [36]. Only Asian species belong to subgenus Macrolespedeza, while both Asian and North American species belong to subgenus lespedeza within the genus Lespedeza. However, these two subgenera are very closely related, and species are indistinguishable. Phylogenetic analysis of Han et al. (2014) [1] showed that L. maritima clustered in the Macrolespedeza group and L. davurica and L. cuneata clustered with the lespedeza group.

Here, we determined the first complete chloroplast genome sequences of subgenus Lespedeza using the Illumina platform and deposited them in the NCBI Genbank. The lengths of complete chloroplast genomes that we obtained were similar to the $\mathrm{cp}$ genome sizes in most of the angiosperms that generally vary from $120 \mathrm{~kb}$ to $160 \mathrm{~kb}$ [39-41]. These $\mathrm{cp}$ genomes showed a quadripartite organization containing a pair of IRs, LSC, and SSC regions as a typical angiosperm [42], and the sequences had similar genome structures, gene orders, and gene contents. However, the higher GC content in the 
IR region of two sequences could be due to the presence of ribosomal RNA in this region [43]. There were no pseudogenes observed in Lespedeza cp genomes, whereas other angiosperms, such as Vernicia fordii, Cynara humilis, and Jacobaea vulgaris, showed pseudogenes in their cp genomes [44,45]. Among 83 protein-coding genes, $p s b L$, rps19, and $n d h D$ showed initiation codons other than the standard ATG in both species. psbL started with TTG, while rps19 and ndhD started with GTG and ATC, respectively. Similar results of a GTG initiation codon in rps19 were also reported in the tobacco chloroplast genome [46]. Further, a TTG initiation codon was reported in the chloroplast inf $A$ gene in the tobacco plant $[47,48]$.

Codon usage bias reflects the importance of molecular evolutionary phenomena such as mutation, selection, and random genetic drift [27]. GC content in codon positions is one of the factors that shape the codon usage biases in different organisms. Typically, the overall GC content for a cp genome of a land plant is low due to the AT-rich intergenic regions [17]. A similar result was observed in the Lespedeza cp genomes. In higher plants, RNA editing occurs during the post-translation process, which means the amino acids change by converting cysteine $(\mathrm{C})$ to uridine $(\mathrm{U})$ at the specific codon. This is an essential mechanism for RNA maturation to avoid incorrect mutations. This process happens when PPR protein (pentatricopeptide repeats) binds to the transcript. RNA editing sites have been identified and validated in many plants including Arabidopsis, tobacco, pea, and tomato [29]. In our study, 84 RNA editing sites were predicted in 35 protein-coding genes of the Lespedeza cp genome. In Ligularia (Asteraceae) cp genomes, 48 RNA editing sites were reported [25]. Serine (S) conversion to leucine $(\mathrm{L})$ was $22 \%$ of the editing, followed by $17 \%$ histidine $(\mathrm{H})$ to tyrosine $(\mathrm{Y})$ conversions. Although RNA editing pattern is conserved in studied Lespedeza cp genomes, detection of RNA editing sites is important to understand the missense mutations of genes.

In this study, SSR distribution was different in each species. In L. davurica and L. cuneata, SSRs were distributed in LSC and SSC region, while in L. maritima, C/G type SSRs in the IR. However, the majority of SSRs were located in the LSC region in all the three species. This was similar to the previously reported cp genome of Cercis chuniana in Fabaceae. Among the SSRs, the highly abundant mononucleotide was the A/T and dinucleotides were mostly composed of AT, suggesting that the SSRs in the cp genomes of the three Lespedeza species were more abundant in AT than in G/C repeats.

Based on mVISTA and sliding window analysis, we identified the sequence divergence regions that could be used as effective molecular markers. Variable regions trnL-trnT, ndhF-rpl32-trnL, and atpA$\operatorname{trnR}$-trnG could be ideal as molecular markers to distinguish the subgenera Lespedeza and Macrolespedeza species. The divergence was distributed in the LSC and SSC regions and mainly associated with the non-coding regions, except for rpl32. Previous studies have shown that the sequence divergence of the IR region was remarkably lower than that of the LSC and SSC regions in many angiosperms [49].

With the development of complete chloroplast sequencing technologies in recent decades, accurate phylogenetic studies have been enhanced, revealing the path to many unknown relationships within the plant kingdom [50]. Therefore, in this study, we used two datasets representing whole chloroplast genomes and 68 protein-coding genes that were present in all cp genomes of Desmodieae. Phylogenetic trees were constructed to determine the phylogenetic position of two Lespedeza (belonging to subgenus Lespedeza) among the species in the Desmodieae. Our results showed a close relationship between these two species and confirmed their phylogenetic position in the Desmodieae. Therefore, we suggest that whole chloroplast genomes and protein-coding genes could be used to find the phylogenetic positions and relationships of Lespedeza in the tribe Desmodieae. According to a previous phylogenetic analysis based on 67 protein-coding genes of cp genomes, L. maritima (belonging to subgenus Macrolespedeza) showed a monophyletic relationship with two genera Campylotropis and Kummerowia. Furthermore, L. maritima showed a sister relationship with Kummerowia, and these two together showed a sister relationship with Campylotropis [14]. Our results also revealed the same relationship between these two genera and resolved the Lespedeza position in the tribe Desmodieae. However, at present, complete chloroplast genomes of many Lespedeza have not been sequenced yet. Those data are needed to distinguish each species and for further investigation of this genus. 


\section{Materials and Methods}

\subsection{Plant Materials and DNA Sequencing}

Fresh leaves of L. davurica and L. cuneata were collected from a rebuild grassland near Yan'an,

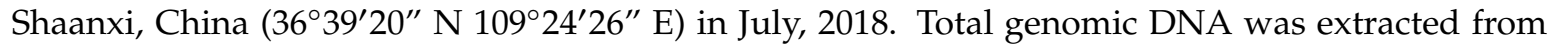
silica-dried leaves using the modified CTAB method [51]. Purified DNA was used for paired-end library preparation according to the manufacturer's protocol (Illumina, San Diego, CA, USA). Genomic DNA was sequenced on the HiSeq X Ten platform (Illumina, San Diego, CA, USA). Complete cp genomes of another six Desmodieae species, namely, Lespedeza maritima (NC_044115), Kummerowia striata (MG867569), Campylotropis macrocarpa (MG867566), Hylodesmum podocarpum (MG867568), Desmodium heterocarpon (MG867567), and Ohwia caudate (MG867572) were downloaded from Genbank.

\subsection{Genome Assembly and Annotation}

High-quality reads were obtained by trimming the raw reads using the Trimmomatic tool [52] and then assembled using the GetOrganelle pipeline (https://github.com/Kinggerm/GetOrganelle/blob/ master/get_organelle_from_reads.py). Subsequently, the assembly was polished by mapping raw reads back to the retrieved sequence, and the consensus sequence was generated using Geneious 10.0.5 software (http://www.geneious.com). We confirmed the low coverage with ambiguous regions by mapped to the reference L. maritima. The consensus sequence was further adjusted by aligning with the available complete cp genomes of Desmodieae. Annotation of all protein-coding sequences and tRNA and mRNA genes were performed using the Dual Organellar GenoMe Annotator (DOGMA) with manual corrections of the uncertain annotations [53]. tRNA genes were confirmed with the tRNAscan-SE program [54]. To determine the accuracy of assembled complete cp genomes, we carried out BLAST using the available sequences of L. davurica and L. cuneata in the NCBI database. We found that these sequences were highly matched with our assembled cp genomes. The circular cp genomes of Lespedeza were drawn using the OrganellarGenome DRAW tool (OGDRAW) [30]. Complete chloroplast genomes of L. davurica and L. cuneata were deposited in Genbank with the accession numbers MH800328 and MN268503 respectively.

\subsection{Codon Usage Bias, RNA Editing Sites, and Sequence Divergence}

Relative synonymous codon usage (RSCU) values and GC contents of protein-coding sequences were calculated by MEGA 6.0 [55]. RNA editing sites of 35 coding sequences of the Lespedeza species were predicted by the online PREP-Cp program with a cutoff value of 0.8 [56]. The mVISTA program [57] was used to align the whole chloroplast genomes of subtribe Lespedezinae. Divergent regions of the chloroplast genomes were determined and variation of nucleotide diversity (Pi) was calculated using the sliding window using DnaSP v5.10. [58] with 200-bp step size and 800-bp window length, which were the common parameters reported in the literature [59-61].

\subsection{Repeat Sequences Analysis}

The MISA [62] microsatellite finder was used to detect SSRs with the parameters set to 10 repeat units for mononucleotide SSRs, 5 repeat units for dinucleotide SSRs, 4 repeat units for trinucleotide SSRs, and 3 repeat units each for tetra-, penta-, and hexanucleotide SSRs. To identify the forward, palindromic, reverse, and complement repeats in three species, REPuter [63] was used.

\subsection{Phylogenetic Analysis}

The whole chloroplast genome sequences of six species belonging to tribe Desmodieae and the out-group Mucuna macrocarpa were downloaded from the NCBI database to determine the phylogenetic positions of the two Lespedeza species analyzed in the present study. Two datasets (complete chloroplast genomes and 68 concatenated protein-coding genes) were used for the analysis. These 68 genes were 
conserved in all nine cp genomes. Coding sequences extracted from each cp genome were aligned using the MAFFT [64] alignment under default parameters, and these alignments were concatenated using Geneious software. Phylogenetic analysis was performed by the maximum likelihood (ML) and Bayesian inference (BI) methods using the final sequence alignments of both datasets. ML trees were constructed with MEGA 6.0 based on the general time reversible (GTR) + I + G model with 1000 replicates. A discrete Gamma distribution was used with four categories. Initial tree(s) for ML were obtained by the neighbor-joining and BioNJ algorithms. BI analysis was conducted using MrBayes 3.2 [65] and a GTR substitution model was used. Two independent Markov chain Monte Carlo (MCMC) chains were run for 1.5 million generations with a subsampling frequency of 200 generations.

\section{Conclusions}

The study presented the complete chloroplast genomes of Lespedeza davurica and L. cuneata. This is the first study reporting the cp genome sequences of Lespedeza subgenus. Our results showed that gene contents, orders, and structures were highly conserved among these species. Results based on codon usage bias and predicted RNA editing sites are useful for further studies about this genus. Our results based on the sequence divergence analysis identified variable regions that can be used for identifying Lespedeza species. Finally, the data obtained from this study could provide a useful resource for further research on these species at the genomic scale and in establishing deeper branches of the phylogeny.

Supplementary Materials: The following are available online at https://www.mdpi.com/2223-7747/9/1/51/s1, Table S1: Summary of gene contents present in the Lespedeza chloroplast genomes, Table S2: Comparison of chloroplast genomes among Desmodieae, Table S3: Summary of relative synonymous codon usage (RSCU) of the codon usage in the Lespedeza cp genomes, Table S4: Predicted RNA editing sites in chloroplast protein-coding genes of Lespedeza, Table S5: Distribution of Simple sequence repeats (SSRs) in the three Lespedeza cp genomes.

Author Contributions: Conceptualization, S.-Q.X., Y.S., and D.-L.G.; methodology, L.Z. and Y.S.; software and formal analysis, Y.S. and D.-L.G.; writing-original draft preparation, Y.S.; writing-review and editing, S.-Q.X.; supervision, S.-Q.X. and W.-Q.W.; funding acquisition, S.-Q.X. and W.-Q.W. All authors have read and agreed to the published version of the manuscript.

Funding: This work was financially supported by the Excellent Doctor Innovation Project of Shaanxi Normal University (S2015YB03), and the Fundamental Research Funds for the Central Universities (GK201902010; GK201702010; GK201701006); The key R \& D plan of Shaanxi province (2018ZDXMSF-079), This work was supported in part by the National Natural Science Foundation of China (No. 61673251, No. 31760101).

Conflicts of Interest: The authors declare that they have no conflict of interest.

\section{References}

1. Han, J.E.; Chung, K.H.; Nemoto, T.; Choi, B.H. Phylogenetic analysis of eastern Asian and eastern North American disjunct Lespedeza (Fabaceae) inferred from nuclear ribosomal ITS and plastid region sequences. Bot. J. Linn. Soc. 2010, 164, 221-235. [CrossRef]

2. Wang, G.H. Differences in leaf $\delta 13 \mathrm{C}$ among four dominant species in a secondary succession sere on the Loess Plateau of China. Photosynthetica 2003, 41, 525-531. [CrossRef]

3. Guan, X.K.; Zhang, X.H.; Turner, N.C.; Xu, B.C.; Li, F.M. Two perennial legumes (Astragalus adsurgens Pall. and Lespedeza davurica S.) adapted to semiarid environments are not as productive as Lucerne (Medicago sativa L.) but use less water. Grass Forage Sci. 2013, 68, 469-478. [CrossRef]

4. Chen, X.L.; An, S.; Liu, S.R.; Li, G.Q. Micro scale spatial heterogeneity and the loss of carbon, nitrogen and phosphorus in degraded grassland in Ordos Plateau, northwestern China. Plant Soil 2004, 259, $29-37$. [CrossRef]

5. Hu, L.; Busby, R.R.; Gebhart, D.L.; Yannarell, A.C. Invasive Lespedeza cuneata and native Lespedeza virginica experience asymmetrical benefits from rhizobial symbionts. Plant Soil 2014, 384, 315-325. [CrossRef]

6. Sharma, B.R.; Rhyu, D.Y. Lespedeza davurica (Lax.) Schindl. extract protects against cytokine-induced $\beta$-cell damage and streptozotocin-induced diabetes. BioMed Res. Int. 2015, 2015, 169256. [CrossRef]

7. Ohashi, H. Nomenclatural changes in Leguminosae of Japan. J. Jpn. Bot. 1982, 57, 29-30.

8. Xu, B.; Gao, X.F.; Wu, N.; Zhang, L.B. Pollen diversity and its systematic implications in Lespedeza (Fabaceae). Syst. Bot. 2011, 36, 352-361. [CrossRef] 
9. Nemoto, T.; Ohashi, H. Seedling morphology in Lespedeza (Leguminosae). J. Plant Res. 1993, 106, $121-128$. [CrossRef]

10. Ohashia, H.; Nemoto, T. A New System of Lespedeza (Leguminosae Tribe Desmodieae). J. Jpn. Bot. 2014, 89, $1-11$.

11. Kim, Y.M.; Lee, J.; Park, S.H.; Lee, C.; Lee, J.W.; Lee, D.; Kim, N.; Lee, D.; Kim, H.Y.; Lee, C.H. LC-MS-based chemotaxonomic classification of wild-type Lespedeza sp. and its correlation with genotype. Plant Cell Rep. 2012, 31, 2085-2097. [CrossRef] [PubMed]

12. Wang, Y.; Zhan, D.F.; Jia, X.; Mei, W.L.; Dai, H.F.; Chen, X.T.; Peng, S.Q. Complete chloroplast genome sequence of Aquilaria sinensis (Lour.) Gilg and evolution analysis within the Malvales order. Front. Plant Sci. 2016, 7, 280. [CrossRef] [PubMed]

13. Zhou, J.; Cui, Y.; Chen, X.; Li, Y.; Xu, Z.; Duan, B.; Li, Y.; Song, J.; Yao, H. Complete Chloroplast Genomes of Papaver rhoeas and Papaver orientale: Molecular Structures, Comparative Analysis, and Phylogenetic Analysis. Molecules 2018, 23, 437. [CrossRef] [PubMed]

14. Jin, D.P.; Choi, I.S.; Choi, B.H. Plastid genome evolution in tribe Desmodieae (Fabaceae: Papilionoideae). PLoS ONE 2019, 14, e0218743. [CrossRef] [PubMed]

15. Yang, M.; Zhang, X.W.; Liu, G.M.; Yin, Y.X.; Chen, K.F.; Yun, Q.Z.; Zhao, D.J.; Almssallem, I.S.; Yu, J. The complete chloroplast genome sequence of date palm (Phoenix dactylifera L.). PLoS ONE 2010, 5, e12762. [CrossRef]

16. Wicke, S.; Schneeweiss, G.M.; Müller, K.F.; Quandt, D. The evolution of the plastid chromosome in land plants: Gene content, gene order, gene function. Plant Mol. Biol. 2011, 76, 273-297. [CrossRef]

17. Yang, J.; Yue, M.; Niu, C.; Ma, X.F.; Li, Z.H. Comparative Analysis of the Complete Chloroplast Genome of Four Endangered Herbals of Notopterygium. Genes 2017, 8, 124. [CrossRef]

18. Parks, M.; Cronn, R.; Liston, A. Increasing phylogenetic resolution at low taxonomic levels using massively parallel sequencing of chloroplast genomes. BMC Biol. 2009, 7, 1-17. [CrossRef]

19. Alkan, C.; Sajjadian, S.; Eichler, E.E. Limitations of next-generation genome sequence assembly. Nat. Methods 2011, 8, 61-65. [CrossRef]

20. Huang, H.; Shi, C.; Liu, Y.; Mao, S.Y.; Gao, L.Z. Thirteen Camellia chloroplast genome sequences determined by high-throughput sequencing: Genome structure and phylogenetic relationships. BMC Evol. Biol. 2014, 14, 1-17. [CrossRef]

21. Daniell, H.; Lin, C.S.; Ming, Y.; Chang, W.J. Chloroplast genomes: Diversity, evolution, and applications in genetic engineering. Genome Biol. 2016, 17, 134. [CrossRef] [PubMed]

22. Yang, X.; Cheng, Y.F.; Deng, C.; Ma, Y.; Wang, Z.W.; Chen, X.H.; Xue, L.B. Comparative transcriptome analysis of eggplant (Solanum melongena L.) and turkey berry (Solanum torvum Sw.): Phylogenomics and disease resistance analysis. BMC Genom. 2014, 15, 412. [CrossRef] [PubMed]

23. Liu, E.; Yang, C.; Liu, J.; Jin, S.; Harijati, N.; Hu, Z.; Diao, Y.; Zhao, L. Comparative analysis of complete chloroplast genome sequences of four major Amorphophallus species. Sci. Rep. 2019, 9, 809. [CrossRef] [PubMed]

24. Yang, A.; Zhang, J.; Yao, X.; Huang, H. Chloroplast microsatellite markers in Liriodendron tulipifera (Magnoliaceae) and cross-species amplification in L. chinense. Am. J. Bot. 2011, 98, e123-e126. [CrossRef]

25. Chen, X.; Zhou, J.; Cui, Y.; Wang, Y.; Duan, B.; Yao, H. Identification of Ligularia Herbs Using the Complete Chloroplast Genome as a Super-Barcode. Front. Pharmacol. 2018, 9, 695. [CrossRef]

26. Song, H.; Liu, J.; Song, Q.; Zhang, Q.; Tian, P.; Nan, Z. Comprehensive Analysis of Codon Usage Bias in Seven Epichloë Species and Their Peramine-Coding Genes. Front. Microbiol. 2017, 8, 1419. [CrossRef]

27. Yang, Y.; Zhu, J.; Feng, L.; Zhou, T.; Bai, G.; Yang, J.; Zhao, G. Plastid Genome Comparative and Phylogenetic Analyses of the Key Genera in Fagaceae: Highlighting the Effect of Codon Composition Bias in Phylogenetic Inference. Front. Plant Sci. 2018, 9, 82. [CrossRef]

28. Wang, W.; Zhang, W.; Wu, Y.; Maliga, P.; Messing, J. RNA editing in chloroplasts of Spirodela polyrhiza, an aquatic monocotelydonous species. PLoS ONE 2015, 10, e0140285. [CrossRef]

29. Wang, M.; Liu, H.; Ge, L.; Xing, G.; Wang, M.; Weining, S.; Nie, X. Identification and Analysis of RNA Editing Sites in the Chloroplast Transcripts of Aegilops tauschii L. Genes 2017, 8, 13. [CrossRef]

30. Lohse, M.; Drechsel, O.; Bock, R. OrganellarGenomeDRAW (OGDRAW): A tool for the easy generation of high-quality custom graphical maps of plastid and mitochondrial genomes. Curr. Genet. 2007, 52, 267-274. [CrossRef] 
31. Powell, W.; Morgante, M.; Mcdevitt, R.; Vendramin, G.G.; Rafalski, J.A. Polymorphic simple sequence repeat regions in chloroplast genomes: Applications to the population genetics of pines. Proc. Natl. Acad. Sci. USA 1995, 92, 7759-7763. [CrossRef]

32. Zhang, Y.; Du, L.; Ao, L.; Chen, J.; Li, W.; Hu, W.; Wei, Z.; Kim, K.; Lee, S.C.; Yang, T.J. The Complete Chloroplast Genome Sequences of Five Epimedium Species: Lights into Phylogenetic and Taxonomic Analyses. Front. Plant Sci. 2016, 7, 306. [CrossRef] [PubMed]

33. Park, I.; Yang, S.; Choi, G.; Kim, W.J.; Moon, B.C. The complete chloroplast genome sequences of Aconitum pseudolaeve and Aconitum longecassidatum, and development of molecular markers for distinguishing species in the Aconitum subgenus Lycoctonum. Molecules 2017, 22, 2012. [CrossRef] [PubMed]

34. Judd, W.S.; Campbell, C.S.; Kellog, E.A.; Stevens, P.F.; Donoghue, M.J. Plant Systematics: A Phylogenetic Approach, 2nd ed.; Sinauer Associates: Sunderland, MA, USA, 2002; pp. 287-292. ISBN 0-87893-403-0.

35. Rahman, A.H.M.M.; Parvin, M.I.A. Study of Medicinal Uses on Fabaceae Family at Rajshahi, Bangladesh. Res. Plant Sci. 2014, 2, 6-8.

36. The Angiosperm Phylogeny Group. An update of the Angiosperm Phylogeny Group classification for the orders and families of flowering plants: APG III. Bot. J. Linn. Soc. 2009, 161, 105-121. [CrossRef]

37. Magalion, S.A.; Sanderson, K.R. Absolute diversification rates in angiosperm clades. Evolution 2001, 55, 1762-1780. [CrossRef]

38. Ohashi, H.; Polhill, R.H.; Schubert, B.G. Desmodieae. In Advances in Legume Systematics. Part 1; Polhill, R.M., Raven, P.H., Eds.; Royal Botanic Gardens, Kew: Richmond, VA, USA, 1981; pp. 292-300.

39. Saski, C.; Lee, S.B.; Daniell, H.; Wood, T.C.; Tomkins, J.; Kim, H.G.; Jansen, R.K. Complete chloroplast genome sequence of Glycine max and comparative analyses with other legume genomes. Plant Mol. Biol. 2005, 59, 309-322. [CrossRef]

40. Wang, W.; Messing, J. High-throughput sequencing of three Lemnoideae (duckweeds) chloroplast genomes from total DNA. PLoS ONE 2011, 6, e24670. [CrossRef]

41. Yi, D.K.; Kim, K.J. Complete chloroplast genome sequences of important oilseed crop Sesamum indicum L. PLoS ONE 2012, 7, e35872. [CrossRef]

42. Diekmann, K.; Hodkinson, T.R.; Wolfe, K.H.; van den Bekerom, R.; Dix, P.J.; Barth, S. Complete chloroplast genome sequence of a major allogamous forage species, perennial ryegrass (Lolium perenne L.). DNA Res. 2009, 16, 165-176. [CrossRef]

43. Qian, J.; Song, J.; Gao, H.; Zhu, Y.; Xu, J.; Pang, X.; Yao, H.; Sun, C.; Li, X.; Li, C.; et al. The complete chloroplast genome sequence of the medicinal plant Salvia miltiorrhiza. PLoS ONE 2013, 8, e57607. [CrossRef] [PubMed]

44. Li, Z.; Long, H.; Zhang, L.; Liu, Z.; Cao, H.; Shi, M.; Tan, X. The complete chloroplast genome sequence of tung tree (Vernicia fordii): Organization and phylogenetic relationships with other angiosperms. Sci. Rep. 2017, 7, 1869. [CrossRef] [PubMed]

45. Doorduin, L.; Gravendeel, B.; Lammers, Y.; Ariyurek, Y.; Chin-A-Woeng, T.; Vrieling, K. The complete chloroplast genome of 17 individuals of pest species Jacobaea vulgaris: SNPs, microsatellites and barcoding markers for population and phylogenetic studies. DNA Res. 2011, 18, 93-105. [CrossRef] [PubMed]

46. Kusnetsov, V.V. Chloroplasts: Structure and Expression of the Plastid Genome. Russ. J. Plant Physiol. 2018, 65, 465. [CrossRef]

47. Shinozaki, K.; Ohme, M.; Tanaka, M.; Wakasugi, T.; Hayashida, N.; Matsubayashi, T.; Zaita, N.; Chunwongse, J.; Obokata, J.; Yamaguchi-Shinozaki, K.; et al. The complete nucleotide sequence of the tobacco chloroplast genome: Its gene organization and expression. EMBO J. 1986, 5, 2043-2049. [CrossRef]

48. Hirose, T.; Ideue, T.; Wakasugi, T.; Sugiura, M. The chloroplast infA gene with a functional UUG initiation codon. FEBS Lett. 1999, 445, 169-172. [CrossRef]

49. Du, Y.; Bi, Y.; Yang, F.; Zhang, M.; Chen, X.; Xue, J.; Zhang, X. Complete chloroplast genome sequences of Lilium: Insights into evolutionary dynamics and phylogenetic analyses. Sci. Rep. 2017, 7, 5751. [CrossRef]

50. Zhang, Y.J.; Ma, P.F.; Li, D.Z. High-throughput sequencing of six bamboo chloroplast genomes: Phylogenetic implications for temperate woody bamboos (Poaceae: Bambusoideae). PLoS ONE 2011, 6, e20596. [CrossRef]

51. Doyle, J.J.; Doyle, J.L. Arapid DNA isolation procedure for small quantities of fresh leaf tissue. Phytochem. Bull. 1987, 19, 11-15.

52. Bolger, A.M.; Lohse, M.; Usadel, B. Trimmomatic: A flexible trimmer for Illumina sequence data. Bioinformatics 2014, 30, 2114-2120. [CrossRef] 
53. Wyman, S.K.; Jansen, R.K.; Boore, J.L. Automatic annotation of organellar genomes with DOGMA. Bioinformatics 2004, 20, 3252-3255. [CrossRef] [PubMed]

54. Lowe, T.M.; Chan, P.P. tRNAscan-SE On-line: Integrating search and context for analysis of transfer RNA genes. Nucleic Acids Res. 2016, 44, W54-W57. [CrossRef] [PubMed]

55. Tamura, K.; Stecher, G.; Peterson, D.; Filipski, A.; Kumar, S. MEGA6: Molecular Evolutionary Genetics Analysis Version 6.0. Mol. Biol. Evol. 2013, 30, 2725-2729. [CrossRef] [PubMed]

56. Mower, J.P. The PREP Suite: Predictive RNA editors for plant mitochondrial genes, chloroplast genes and user-defined alignments. Nucleic Acids Res. 2009, 37, W253-W259. [CrossRef]

57. Frazer, K.A.; Pachter, L.; Poliakov, A.; Rubin, E.M.; Dubchak, I. VISTA: Computational tools for comparative genomics. Nucleic Acids Res. 2004, 32, W273. [CrossRef]

58. Librado, P.; Rozas, J. DnaSP v5: A software for comprehensive analysis of DNA polymorphism data. Bioinformatics 2009, 25, 1451-1452. [CrossRef]

59. Chen, X.; Cui, Y.; Nie, L.; Hu, H.; Xu, Z.; Sun, W.; Gao, T.; Song, J.; Yao, H. Identification and Phylogenetic Analysis of the Complete Chloroplast Genomes of Three Ephedra Herbs Containing Ephedrine. BioMed Res. Int. 2019, 2019, 5921725. [CrossRef]

60. Cui, Y.; Nie, L.; Sun, W.; Xu, Z.; Wang, Y.; Yu, J.; Song, J.; Yao, H. Comparative and Phylogenetic Analyses of Ginger (Zingiberofficinale) in the Family Zingiberaceae Based on the Complete Chloroplast Genome. Plants 2019, 8, 283. [CrossRef]

61. Zhao, Z.; Wang, X.; Yu, Y.; Yuan, S.; Jiang, D.; Zhang, Y.; Zhang, T.; Zhong, W.; Yuan, Q.; Huang, L. Complete chloroplast genome sequences of Dioscorea: Characterization, genomic resources, and phylogenetic analyses. PeerJ 2018, 6, e6032. [CrossRef]

62. Beier, S.; Thiel, T.; Münch, T.; Scholz, U.; Mascher, M. MISA-web: A web server for microsatellite prediction. Bioinformatics 2017, 33, 2583-2585. [CrossRef]

63. Kurtz, S.; Schleiermacher, C. REPuter: Fast computation of maximal repeats in complete genomes. Bioinformatics 1999, 15, 426-427. [CrossRef] [PubMed]

64. Katoh, K. MAFFT: A novel method for rapid multiple sequence alignment based on fast Fourier transform. Nucleic Acids Res. 2002, 30, 3059-3066. [CrossRef] [PubMed]

65. Ronquist, F.; Teslenko, M.; van der Mark, P.; Ayres, D.L.; Darling, A.; Höhna, S.; Larget, B.; Liu, L.; Suchard, M.A.; Huelsenbeck, J.P. MrBayes 3.2: Efficient Bayesian phylogenetic inference and model choice across a large model space. Syst. Biol. 2012, 61, 539-542. [CrossRef] [PubMed] 